\title{
EVIDENCIAS DE AGROASTRONOMÍA ENTRE LOS ANTIGUOS MAYAS
}

EVIDENCES OF AGRO-ASTRONOMY AMONG THE ANCIENT MAYAS

\author{
SusAn Milbrath \\ Florida Museum of Natural History
}

\begin{abstract}
Resumen: El estudio se enfoca en un almanaque agrícola del Posclásico maya que figura en el Códice Madrid, 12b-18b, el cual parece mostrar registros de eclipses integrados con observaciones de Venus en el contexto del calendario de 260 días (Tzolkin). Usando las fechas de eclipses visibles en Yucatán es posible fechar los eventos entre los años de 1452 y 1492, contemporáneos al uso de este códice.

Palabras clave: Códice Madrid, ciclos agrícolas, Venus, eclipses solares, calendario Tzolkin.

ABSTRACT: This study focuses on a Postclassic Maya agricultural almanac in the Madrid Codex (12b-18b), which seems to show a registry of eclipses integrated with observations of Venus in the context of a 260-day calendar (Tzolkin). Using the dates of eclipses visible in Yucatan, it is possible to date the events between 1452 and 1492, contemporary with the use of the codex.
\end{abstract}

KeYwords: Madrid Codex, agricultural cycles, Venus, solar eclipses, Tzolkin calendar.

RECEPCIÓn: 24 de noviembre de 2014.

ACEPTACIÓN: 15 de mayo de 2015. 



\title{
EVIDENCIAS DE AGROASTRONOMÍA ENTRE LOS ANTIGUOS MAYAS
}

\author{
SuSAN MiLbRath \\ Florida Museum of Natural History
}

En un almanque agrícola del Códice Borgia, documento del Posclásico Tardío procedente del Centro de México, que exhibe paralelos con el Códice Madrid (Aveni, 1999; Bricker 2010; Vail y Aveni, 2004; Hernández y Vail, 2010), se aprecian vínculos entre Venus y los eclipses (Milbrath, 2013). Una imagen en la tabla de eclipses del Códice Dresden (58b) muestra claramente la importancia de Venus en el contexto de los ciclos de eclipses también en el área maya (Milbrath, 1999: 162). Lo más probable es que el Códice Madrid se feche a mediados del siglo xv, en el Posclásico Tardío, al igual que el Códice Borgia (Vail y Aveni, ibid.: 12). El almanaque de 260 días, con imágenes de eclipses en el Códice Madrid, 12b-18b (figuras 1 y 2), ha sido comparado con uno que aparece al principio del Códice Borgia, el cual muestra el calendario completo de 260 días (Bricker y Bricker, 2015: 172; Just, 2004). El calendario de 260 días está organizado en cinco filas de 52 días en el Borgia, mientras que en el Madrid tiene una disposición en cuatro filas de 65 días, y las últimas trecenas en cada fila están ausentes, ya sea porque el espacio era insuficiente, ya porque la trecena faltante pudiera encontrarse en la página $12 \mathrm{~b}$, que precede al Tzolkin.

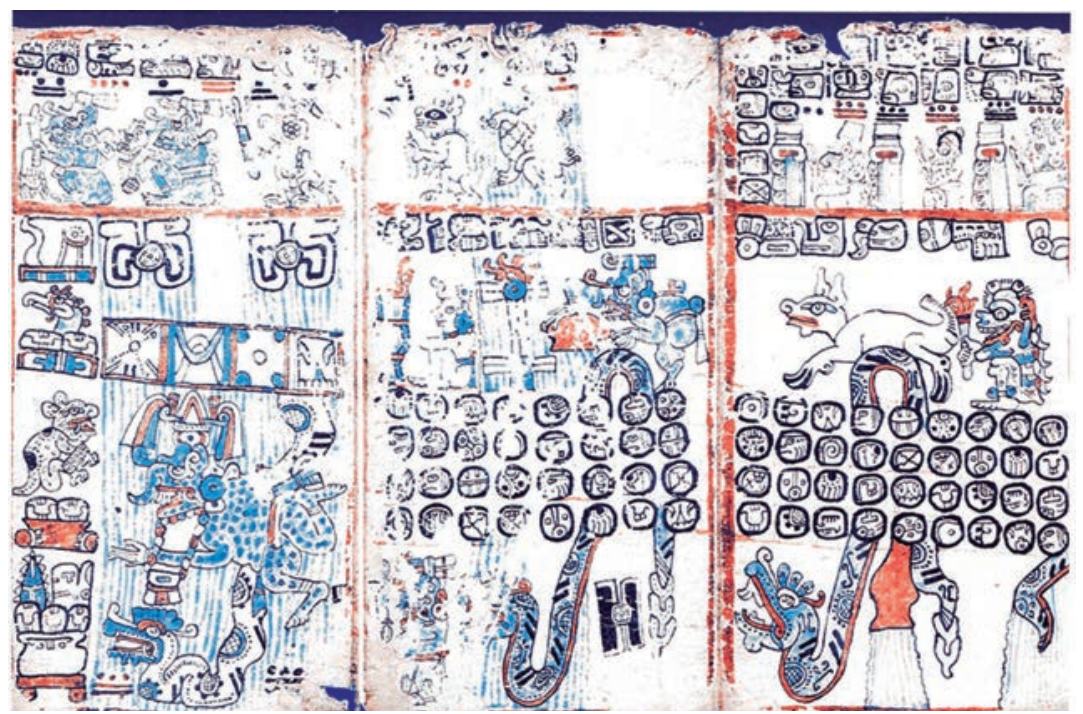

FIGURA 1. Códice Madrid, 12b-15b (apud Milbrath, 1999). 

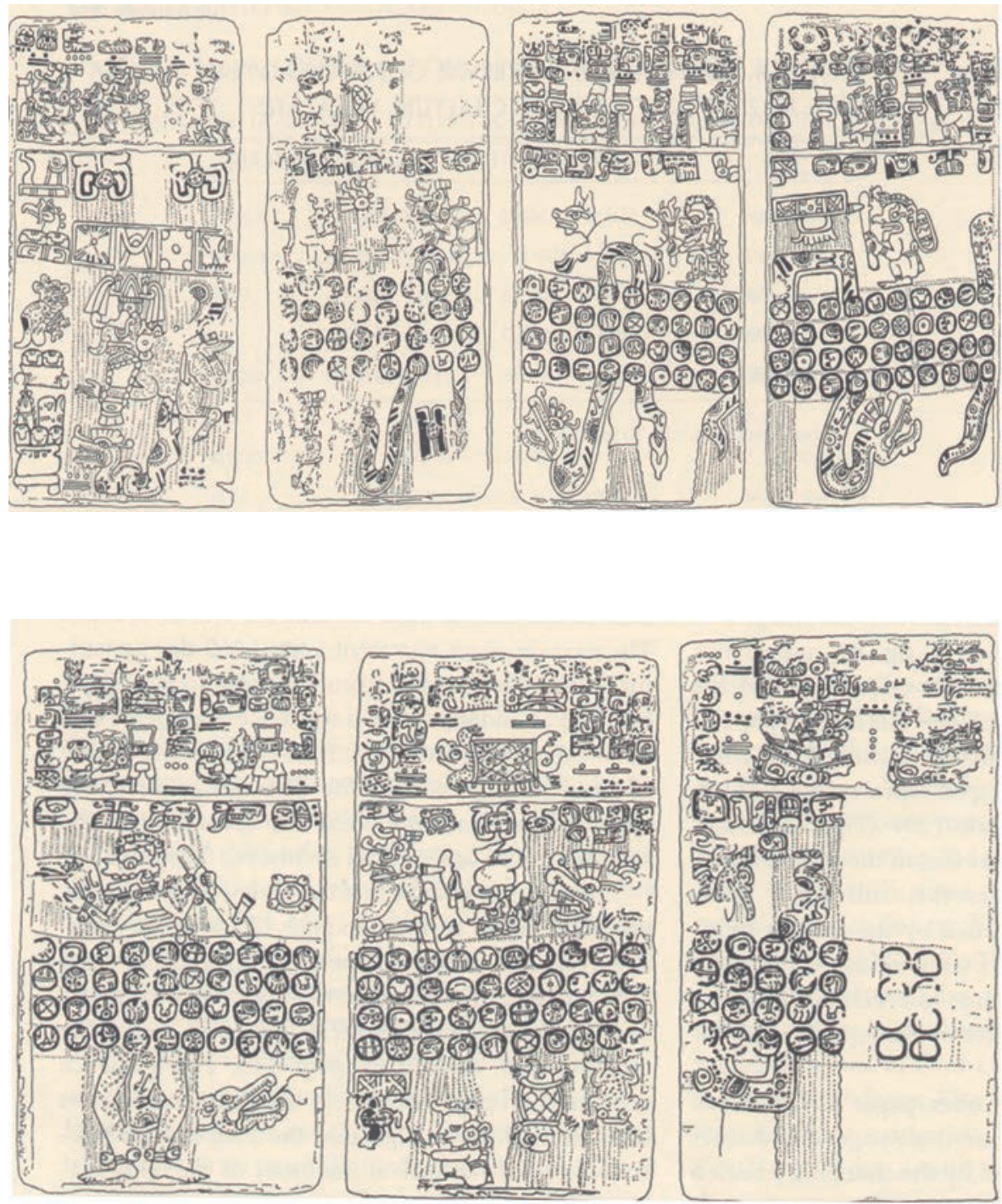

Figuras 2a y 2b. Códice Madrid, 12b-18b (adaptado de Milbrath, 1999: figura 7.3). 
En su libro Astronomy in the Maya Codices, Harvey y Victoria Bricker (2011: 566569) anotan que el número uno inicia cada una de las cuatro filas del Tzolkin expuestas en el Códice Madrid (13b). Su modelo propone que el número 13 en negro en la página $12 \mathrm{~b}$ marca el intervalo de la primera trecena para las primeras cuatro filas que siguen en las páginas $13 \mathrm{~b}-18 \mathrm{~b}$ (figura 1 ).

Los Bricker reconocen imágenes de las estaciones del año en este almanaque del Códice Madrid, y anotan que serían necesarios tres cursos del almanaque de 260 días en los años 924-926 d.C. para contar con todos los símbolos y glifos de eclipses (12b, 13b y 17b) y otras imágenes en páginas individuales. Aunque enfatizan la importancia de tales símbolos de eclipses en el almanaque del Códice Madrid, sorprendentemente no vinculan los eclipses a los observables en Yucatán, donde se originó dicho códice. Asimismo, identifican un grupo de eclipses en la página 13, reunidos al inicio de la temporada agrícola (marzo-abril), y un segundo grupo en septiembre-noviembre, en la página 17 (Bricker y Bricker, 2011: tablas 11-17).

La idea de que el almanaque se traslapa con la temporada agrícola está generalmente en concordancia con las investigaciones que he publicado antes, pero hay algunas diferencias en los detalles relacionados con las estaciones. Por ejemplo, los Bricker hablan del venado macho sin cuernos en la página $14 \mathrm{~b}$ como un signo del período que va desde marzo hasta mayo, mientras que esta imaginería es más limitada en el tiempo, basada en los datos biológicos que especifican que marzo es la época cuando los venados pierden sus astas en Yucatán (Milbrath, 1999: 61; Leopold, 1959). La razón por la cual ellos identifican la imagen del venado como una que se aplica al mes de mayo es porque la página $14 \mathrm{~b}$ debe corresponder a dicho mes en una de las corridas del almanaque (Bricker y Bricker, 2011: tablas 11-19). La posición estacional cambiante de algunas páginas en varios cursos a través del almanaque está en desacuerdo con mi interpretación, como discutiré más adelante.

Los Bricker argumentan también que el almanaque del Códice Madrid, 12b-18b, incorpora "predicciones" de eclipses que datan del inicio del siglo x, usando múltiples cursos del almanaque para dar cuenta de su posición en el año. Su evidencia más importante para este fechamiento temprano del almanaque se encuentra al comparar fechas de dos almanaques estacionales en el Códice Madrid, 10-11, que se corresponden cercanamente con un almanaque estacional presente en el Códice Dresden (Bricker y Bricker, 2011: figuras 11-40; tablas 11-15 y 11-18). Sobre esta base, concluyen que las fechas del siglo x convienen mejor a estos dos almanaques estacionales y al almanaque del Tzolkin en las páginas $12 \mathrm{~b}-18 \mathrm{~b}$. Asimismo, identifican dos referencias al Haab en la banda augural en el Códice Madrid, 14b$15 b$, que datan en el 925 d.C., si bien ubican a todos los otros almanaques del Códice Madrid entre 1451 d.C. y 1488 d.C. ${ }^{1}$ En ausencia de inscripciones de Cuen-

\footnotetext{
${ }^{1}$ Las fechas del Haab del siglo x están basadas en el trabajo de Vail y V. Bricker (2004: tabla 7.1), quienes notaron que los almanaques en el Madrid (14b-15b) pueden registrar una variante para el glifo
} 
ta Larga o registros de ciclos de katun que colocarían estas referencias aisladas como fechas del siglo $\mathrm{x}$, es difícil aceptar una fecha tan temprana cuando ellos mismos datan en el siglo xv los otros almanques contenidos en el Códice Madrid. Uno podría preguntarse ¿por qué incluir un predictor de eclipse tan anticuado en un códice que generalmente se fecha en el siglo xv? Más aún, ¿cómo sabían los sacerdotes que usaban este almanaque en el siglo xv que se trataba de un período de más de 500 años previos? Tales preguntas me llevaron a explorar otra alternativa: la de que el almanaque del Códice Madrid sea un registro Posclásico de eclipses observados en Yucatán.

Con el advenimiento de un creciente interés en eventos en los códices que pertenecen a fechas actuales se ha desarrollado una línea de investigación, iniciada por Aveni (op. cit.) y Victoria Bricker (2001) con el estudio del Códice Borgia, y seguida por Harvey y Victoria Bricker (2011) en su trabajo sobre el Códice Dresden. Por mi parte, estudié el Códice Madrid, 12b-18b, para ver si los eclipses podrían haber sido registrados como eventos ya vistos en Yucatán. También exploré si los eventos observables de eclipses pueden correlacionarse con eventos de Venus, un enfoque que se mostró exitoso en mi estudio de la secuencia narrativa en el Códice Borgia (29-46), publicado en 2013.

En mi investigación actual utilizo seis premisas que concuerdan con las interpretaciones de los Bricker:

1) El período representado es de 260 días, como indica in extenso el almanaque que figura en el Códice Madrid, 13b-18b, el cual al parecer comienza con un intervalo de 13 días, registrado en negro en $12 \mathrm{~b}$.

2) La página $12 \mathrm{~b}$ introduce el almanaque in extenso, y el número 13, en rojo en esta página, se refiere a una fecha del Tzolkin que precede a la primera columna de fechas que siguen inmediatamente (trazado en figura 1).

3) La banda augural en 13b-18b alude a los eventos representados en las páginas correspondientes.

4) La imaginería se traslapa con la temporada agrícola y al menos parte de la secuencia muestra la estación lluviosa, claramente representada por el fondo de lluvia en una serie de páginas (12, 13 y 15-18).

5) Dado que están representados múltiples símbolos de eclipses, la imaginería debe representar a su vez múltiples eventos de eclipses.

6) Las fechas del Tzolkin en los períodos de trecena representadas en páginas individuales con símbolos de eclipses coordinan eclipses verdaderos visibles en alguna parte del mundo.

He agregado cuatro premisas propias que difieren notablemente del modelo publicado por los Bricker en 2011:

Uo y una escritura fonética de Cumku, las cuales se relacionan con fechas del Tzolkin en la misma página, para reconstruir las fechas de la Rueda Calendárica, que datan en enero y diciembre de 925. 
1) La serpiente Chicchan representa la versión maya de Quetzalcóatl, y ambas son serpientes emplumadas que figuran a Venus.

2) La representación de los cascabeles en la serpiente Chicchan remite a las Pléyades, las cuales eran llamadas en maya $t z a b$, en alusión al cascabel de la serpiente.

3) La secuencia de fechas de eclipse data del Posclásico Tardío, cuando el códice fue pintado, porque el almanaque es un registro en tiempo real de eventos en la historia natural, y no un documento que predecía eclipses.

4) La secuencia de eclipses incorpora los eclipses del Posclásico Tardío visibles en Yucatán, una premisa que claramente distingue el presente estudio de la investigación publicada por los Bricker, ya que los eclipses a los cuales ellos hacen referencia en el siglo x temprano (924-926 d.C.) no fueron visibles en Yucatán.

Inicio con la primera premisa, que hace necesaria una discusión para mostrar cómo mi interpretación de las serpientes Chicchan difiere de la posición tomada por los Bricker, quienes siguen a Brian Just (op. cit.: 262, 274) en la identificación de las serpientes como un mecanismo para dividir el calendario en trecenas, como se observa en el Códice Borgia, 72, con cuatro ofidios con 12 espaciadores circulares marcando los períodos de trecenas. Uno de los problemas de este enfoque es que, aunque la división de puntos de las trecenas preservadas en cada fila está marcada por serpientes Chicchan, en verdad hay dos serpientes en las cuatro primeras filas de trecenas en el Códice Madrid, 13b-14b (figura 2). Además, estas sierpes no son simples marcadores calendáricos, sino que juegan un papel mucho más importante. En Star Gods of the Maya indiqué que en recuentos etnográficos mayas se narra que la serpiente Chicchan trae la lluvia (Milbrath, 1999: 36). Como anoté allí, los atributos cambiantes de Chicchan, algunos con cascabeles y otros sin ellos, pueden ser significativos en términos de la aparición y desaparición de las Pléyades, llamadas en Yucatán $t z a b$, esto es, "el cascabel de la serpiente cascabel," lo cual tiene mucho que ver con el papel que dichas constelaciones juegan en el ciclo de las lluvias (Milbrath, op. cit.: 261). Las representaciones pluviales y de Chaac, el dios de la lluvia, indican que el almanaque concierne a estaciones pluviales. Propongo que la serpiente Chicchan asociada con la lluvia, contraparte de Quetzalcóatl en México central (Taube, 1992: 140, figura 76e), representa a Venus. El interés por Venus figura también en las bandas celestiales con quincunces en las páginas 12 y 15, un símbolo encontrado en la imaginería de Venus en el centro de México (Milbrath, 1999: figura 5.6b). Y el Dios H, una versión antropomorfa de Quetzalcóatl identificada por Karl Taube (op. cit.), también aparece sentado en la página 13 donde empieza el Tzolkin en el almanaque del Códice Madrid (así como en la página 17).

El ciclo estacional mostrado en el Códice Madrid, 13b-18b, está claramente integrado con eclipses, como los Bricker notaron, pero Venus también está representado en el Dios $\mathrm{H}$ y en la serpiente Chicchan, y hay un patrón implícito en las sierpes con las colas de cascabel. Este patrón que se repite, colocando a Venus cerca de las Pléyades al inicio de la estación agrícola, puede ser documentado 
por más de dos mil años (Milbrath, 1999: 210). De hecho, que Venus pase por las Pléyades en marzo y abril puede ser representado por la serpiente Chicchan que porta cascabeles en el principio de la secuencia (Madrid, 13-14). No hay duda de que el posicionamiento de Venus cerca de las Pléyades se convirtió en un marcador para el inicio de la estación agrícola.

Existen otras referencias más obvias a las estaciones en las imagenes y los glifos en la banda augural del Códice Madrid, 13b-18b (op. cit.: 62). Los glifos de fuego que aparecen en la primera mitad de la secuencia de la banda augural (Madrid, 13b-16b) representan la quema de los campos de labor, la cual comienza en febrero, al inicio de la temporada agrícola en Yucatán. La leña que arde en la página 13 también simboliza el principio de dicha quema en febrero. En la página 14 , una antorcha que aparece al lado del venado macho muestra el sacrificio de un venado al fuego en el festival de Mac, que sabemos tenía lugar en marzo, según el calendario de Diego de Landa (Tozzer, 1941). El venado macho sin astas es asimismo un símbolo biológico del mes de marzo en Yucatán, como se ha anotado antes. Y según el calendario de festivales de Landa, la divinidad que carga un bulto (Madrid, 15) es el dios mercader que se honra en Muan, celebrado en mayo alrededor de la época de la conquista.

Propongo que estas observaciones estacionales se realizaron en el contexto de un almanaque que muestra los eclipses solares y la posición de Venus en las diferentes estaciones. ${ }^{2}$ De hecho, parece que este documento es un registro de eventos astronómicos contemporáneos al tiempo en que el códice fue pintado, en el siglo xv. Para determinar exactamente lo que son estos eventos, enfoqué mi investigación en las posiciones de Venus y los eclipses solares en Yucatán durante el período Posclásico Tardío, desde 1300 a 1520, el rango más amplio de fechas que puede ser asociado con el tiempo en que se pintó el Códice Madrid.

Comencemos con los eventos de eclipses, entre los cuales los más significativos fueron seguramente los totales. Durante el Posclásico Tardío encontramos sólo cuatro eclipses totales visibles en Yucatán con una alta magnitud $(0.90 \mathrm{o}$ más): el 16 de julio de 1311 (magnitud local de 0.97), el 17 de junio de 1452 (magnitud local de 0.96), el 13 de abril de 1325 y el 8 de agosto de 1496 (magnitud local de 0.96). Cinco más fueron eclipses del tipo híbrido (hybrid): 15 de junio en 1303 (magnitud local de 0.92), 26 de agosto de 1318 (magnitud local de 0.99), 27 de septiembre de 1372 (0.93), 26 de julio de 1405 (magnitud local de 1.01) y 13 de febrero de 1477 (magnitud local de 0.99). ${ }^{3}$ Este tipo de eclip-

\footnotetext{
${ }^{2}$ Según mi modelo publicado en 1999, el ciclo estacional está de alguna manera integrado con eclipses representados en las páginas 13 y 17, pero no intenté posicionar eclipses en la secuencia en mi libro, anotando únicamente que los intervalos representados en las páginas podrían ser apropiados para eclipses si es que cada página representara un período que fuera designado por el número de signos de día (Milbrath, 1999: 61). Este modelo fue sólo un intento preliminar para incorporar el ciclo de eclipses, el cual es tratado en este estudio, de carácter más definitivo.

${ }^{3}$ Espenak y Meeus (2006: 4) anotan el tipo de eclipse y apuntan que "eclipse magnitude is always less than 1.0 for partial and annular eclipses, but equal to or greater than 1.0 for total and hybrid eclip-
} 
ses son de especial interés, pero dado que en el almanaque se representan varios eclipses, aquellos que sucedieron en pares durante el mismo año pueden ser de mayor interés, aunque hay sólo dos años en que ambos eclipses fueron visibles en Yucatán y apenas un año (1340) en que los dos tuvieron una magnitud local superior a 0.50 (tablas 1 y 2). Los pares de eclipses están siempre separados por cinco o seis meses, relacionados con intervalos de 148 días o 177 días, registrados en las tablas de eclipses del Códice de Dresden (Milbrath, 1999: lámina 3). Usando nuestro calendario, que corre de enero a diciembre, en vez del año maya comenzando con Pop, la tabla 1 muestra 19 años con pares de eclipses durante el siglo xiv que fueron visibles en Yucatán con una magnitud de más del $50 \%(0.50)$. La tabla 2 incluye un número mayor de pares de eclipses entre 1400 y 1520 , mostrando 24 años con pares de eclipses, y tres años (1405, 1470 y 1516) que supieron de tres eclipses solares. Entre 1300 y 1520, 15 eclipses solares fueron visibles en Yucatán con una magnitud alta de 0.90 o más $(1301,1303,1311,1318$, 1325, 1355, 1372, 1383, 1402, 1405, 1434, 1452, 1477, 1481 y 1496). ¿Cuál de los eclipses anotados en las tablas 1 y 2 tiene más probabilidades de haber sido el representado? Para responder a esto retornemos a la discusión acerca de la serpiente de Venus en el contexto del ciclo agrícola.

Dada la prominencia de la serpiente Chicchan representando a Venus en el almanaque, y el período de 260 días iniciado por el almanaque del Tzolkin, los eclipses totales en 1452 y 1496 son de especial interés, ya que los pares de eclipses en estos años coincidieron con un tiempo a lo largo del cual Venus fue visible por alrededor de 260 días, durante un período que cubriría el ciclo agrícola completo, desde la preparación del terreno para la siembra del maíz, hasta su cosecha, tomando en cuenta que hay variabilidades en Yucatán que tienen que ver con el tipo de maíz empleado (Terán y Ramussen, 1994). El ciclo largo agrícola, de 260 días, todavía se preserva en varias comunidades mayas actuales (Milbrath, 1999: 59).

En 1496 Venus fue la Estrella Matutina desde el inicio de enero hasta septiembre, mientras que en 1452 fue visible por un período de tiempo similar pero como Estrella Vespertina. ${ }^{4}$ Las tablas 1 y 2 muestran que en años de eclipses en pares, la visibilidad de la Estrella Matutina se traslapa con el ciclo agrícola desde el inicio de febrero hasta el principio de la cosecha, en octubre, dos veces en el siglo xiv (1376 y 1384), mientras que en la centuria siguiente la Estrella Vespertina se observó tres veces durante este mismo período (1452, 1484 y 1492).

ses"). Las fechas y magnitud local de los eclipses solares viene de Verbelen (2003), quien da la magnitud local en distintos sitios arqueológicos. En las tablas 1 y 2 uso los datos de Uxmal, porque el Códice Madrid probablemente viene de Mayapán, que no se anota en Verbelen, pero queda cerca de Uxmal.

${ }^{4}$ Los Bricker (op. cit.) favorecen la colocación del almanaque en un período en que Venus era la Estrella Matutina, argumentando que el glifo de una figura sentada en la página 17b nombra la misma tortuga que fue víctima de la Estrella Matutina saliente en el Códice Dresden, 49, en la tabla de Venus. Esto les hace proponer que el Códice Madrid, 17b, corresponde a la salida de la Estrella Matutina, pero también podría simplemente nombrar a la tortuga (ac), representada en el Códice Madrid, 17b (Milbrath, 1999: 176). 
Para poder colocar los eventos astronómicos en tiempo real, también exploré si las fechas para eventos de eclipses correspondían a las fechas del Tzolkin que aparecen en aquellas páginas del Códice Madrid que muestran símbolos de eclipses (esto es, 12b, 13b y 17b). Para la página 12b estas fechas pueden caer en cualquiera de las trecenas que preceden inmediatamente las cuatro trecenas que comienzan en la página 13 con las fechas $1 \mathrm{Imix}, 1 \mathrm{Cimi}, 1$ Chuen, $1 \mathrm{Cib}$, de acuerdo con el modelo publicado por los Bricker en 2011. Dicho modelo relaciona eventos de eclipses con las fechas del Tzolkin en la trecena que comienza en una página con un glifo de eclipse, pero algunas veces las fechas apropiadas quedan en la siguiente página, porque las trecenas individuales generalmente se extienden a través de una página y media (Bricker y Bricker, 2011: figuras 11-52 a 11-55). De esta manera, ellos apuntan correspondencia si es que un eclipse cae en alguna trecena que comience en las páginas con un glifo de eclipse, lo cual parece justificado en el caso de la página $12 \mathrm{~b}$, porque un período de trecena completo parece estar representado en esa página. Para las páginas 13 y 17, sin embargo, uso una ventana temporal más precisa, ya que las fechas son consideradas como correspondientes con eventos de eclipses sólo cuando la fecha de Tzolkin es registrada en la página con un símbolo de eclipse. Al revisar la tabla 1, del siglo xiv, vemos que siete de 20 eclipses corresponden a fechas del Tzolkin registradas en las páginas con símbolos de eclipse, y seis de esas fechas se refieren a eclipses que fueron visibles en Yucatán, y están consignadas en letras negritas. Las otras fechas en negritas son eclipses visibles en Yucatán, pero que no corresponden a fechas del Tzolkin anotadas en páginas con símbolos de eclipses. La tabla 2 muestra incluso una mayor correspondencia. En el período entre 1400 y 1520, 15 de 33 eclipses caen en fechas de Tzolkin que están en páginas con símbolos de eclipse, y 12 de éstas son eclipses que fueron visibles en Yucatán, en fechas señaladas también con negritas. ${ }^{5}$

Investigué asimismo si la página introductoria pudiera ser una fecha de eclipse específica, representada por el número 13 en rojo, que sirve como un coeficiente del Tzolkin. En la página 12b, dicho número podría referirse a cualquiera de las cuatro fechas de Tzolkin que preceden las cuatro fechas marcadas con el número uno en la página 13b (aunque apenas el primero está bien conservado: 1 Imix). En este caso, sólo cuatro fechas de Tzolkin pueden ser candidatas: 13 Ahau, 13 Chicchan, 13 Oc y 13 Men. Las tablas 1 y 2 indican que sólo uno de los eclipses tiene una de ellas con el número 13 (1395), pero no fue un eclipse visible en Yucatán. Es posible que la fecha 1 Cimi para dos de los eclipses (1452 y 1485) fuera en verdad 13 Chicchan, si es que el "nuevo día" comenzaba al mediodía. ${ }^{6}$ La tabla 2 muestra que en el año 1452 hubo un eclipse observado en la mañana

${ }^{5}$ La Tabla 2 cubre hasta el final de 1520, pero no hay pares relevantes de eclipses entre 1511 y 1520 .

${ }^{6}$ Thompson (1960: 102) favorece el amanecer como el comienzo del día en los tiempos del Clásico maya, pero anota que esto no es conclusivo en manera alguna. Indica que en Chan Kom hay evidencia de conceptuarse a un día como corriendo de un mediodía al siguiente. 
con una fecha 1 Cimi, que podría haber sido fechado para 13 Chicchan. ${ }^{7}$ El eclipse total de 1452, registrado en junio, es de gran interés, pues tuvo un paso de totalidad sobre Yucatán (0.96 de magnitud).

Se puede suponer que la secuencia comenzara en la página 12b con un eclipse total visible el 17 de junio de 1452, que hubiera tenido la fecha 13 Chicchan si el día comenzaba al mediodía. Esta fecha puede haber sido representada literalmente en esta página por el número 13 en rojo, a la par de la cabeza de la serpiente Chicchan. La página $12 \mathrm{~b}$, entonces, conmemoraría un eclipse total que ocurrió cinco días después del solsticio de verano (12 de junio del calendario juliano y 21 de junio del gregoriano), seguido de un segundo eclipse en diciembre, posicionado precisamente en el solsticio de invierno (tabla 2, 11 de diciembre, juliano), aunque el segundo no fue visible en Yucatán. Los eclipses en pares en junio y diciembre se relacionan con la imaginería en el Códice Madrid, 12b, ya que el par de glifos de eclipses está suspendido sobre una banda celestial, que sirve como un marcador para el punto de intersección de la Vía Láctea y la eclíptica en el "zodíaco" del Códice París, donde el sol está posicionado en los solsticios (Milbrath, 1999: figura 7.6a). El par de símbolos de eclipse sobre la banda celestial en el Códice Madrid, 12b, puede simbolizar un par de eclipses que tuvieron lugar en o cerca de los solsticios en 1452, y esta página también puede servir como una introducción a observaciones subsecuentes de eclipses en relación con el ciclo estacional.

Regresando a la serpiente Chicchan como representación de Venus, vale la pena anotar que fue la Estrella Vespertina desde febrero hasta octubre en 1452 , como se ve en la primera columna que marca los eventos de Venus en la tabla 2. Esto representa un período de alrededor de 260 días, que cubriría la temporada agrícola en Yucatán. No sólo Venus fue visible como Estrella Vespertina a través de la temporada agrícola, sino que este patrón se repetiría ocho años más tarde en 1460, y en intervalos similares después en 1468, 1476, 1484 y 1492 (los últimos dos años incluyeron eclipses visibles en Yucatán, tabla 2). Las Pléyades también estarían junto a Venus en su posición de Estrella Vespertina, ya que esta configuración sólo podía ser vista en el cielo occidental al principio de la temporada agrícola (Milbrath, op. cit.: 210).

La página $12 \mathrm{~b}$ puede referirse a un par de eclipses específicos en 1452, ambos vinculados con los solsticios, y el siguiente Tzolkin in extenso, en las páginas $13 \mathrm{~b}$ a $18 \mathrm{~b}$, se referiría entonces a las observaciones de Venus como la Estrella Vespertina visible por alrededor de 260 días, desde febrero hasta octubre. Si la página $12 \mathrm{~b}$ introduce el almanaque que tiene las observaciones de eclipses emparejados y Venus en el contexto del ciclo agrícola, entonces las páginas 13b a 18b

\footnotetext{
${ }^{7}$ En la tabla 2 las fechas siguen la convención de que el día comienza a medianoche, pero si el día comenzara a mediodía, cualquier eclipse que caiga antes de las 12:00, sería registrado tal vez un día antes en el Tzolkin. En este caso, los eclipses en el día 1 Cimi estarían fechados para 13 Chicchan, una fecha en la página 12, lo que sería el caso para los eclipses totales en junio 17 de 1452 y 16 de marzo de 1485 .
} 
que siguen pueden representar una sola corrida del almanaque de 260 días en un año, con un patrón similar que se encuentra a un intervalo de ocho años, y tal vez el almanaque se usaba cada ocho años. Estas observaciones podrían coincidir con el ciclo agrícola que comienza con la quema del campo en febrero y termina en octubre con la cosecha (aunque esto puede continuar más allá, Milbrath, 1999: 13-14). Por ejemplo, el maíz llamado xnuk nal se siembra de mayo a julio, y la cosecha empieza en agosto, si bien madura en octubre (Terán y Ramussen, op. cit.: 206-207, cuadro 67).

La tabla 2 muestra que en intervalos de ocho años durante el siglo xv, la primera aparición de la Estrella Vespertina (V-primera) tuvo lugar en febrero, al principio de la temporada agrícola, un punto de inicio del ciclo de maíz que, como apunté, todavía se preserva en muchas comunidades mayas en la península de Yucatán. La primera aparición de la Estrella Vespertina corresponde a febrero en los años 1452, 1460, 1468, 1476, 1484 y 1492, de los cuales sólo tres (1452, 1484 y 1492) registraron eclipses visibles en Yucatán.

El enfoque en un patrón repetitivo de los eclipses solares vinculados con Venus como Estrella Vespertina con el ciclo de siembra es claramente una forma de agroastronomía que apenas estamos comenzando a investigar. Hoy en día los campesinos observan a Venus en mayo para hacer pronósticos acerca del ciclo agrícola (Alfonso Morales, com. pers., 2013).

A la luz de los nuevos estudios sobre los eventos en tiempo real en los códices de Mesoamérica, mi modelo sugiere que el almanaque del Códice Madrid registra un patrón repetitivo que enlaza a Venus, los eclipses y el ciclo agrícola. Este registro parece cubrir un período que va de 1452 a 1492 . Asociar el almanaque al ciclo de Venus como la Estrella Vespertina también incorpora el período en que pasó por las Pléyades, como lo hizo desde marzo hasta el inicio de abril en 1452. La serpiente de Venus, portando sus cascabeles al principio de la secuencia en las páginas $13 \mathrm{~b}$ a $14 \mathrm{~b}$, sugiere que tales imágenes están fechadas para el ciclo agrícola temprano, una posición confirmada por otras imágenes estacionales en estas páginas y las que les siguen. En apariencia, al juntarse Venus con las Pléyades al principio de la estación agrícola, se esperaban lluvias y una mayor producción de lo sembrado. ${ }^{8}$ Este vínculo entre la Estrella Vespertina y la fertilidad agrícola pareciera confirmar los patrones investigados por Ivan Šprajc $(1993,1996)$, quien nota que la Estrella Vespertina es muchas veces asociada con la fecundidad. También sugiere que la serpiente emplumada, con su cola de cascabel, puede referir a una imagen de Venus estacional en contextos agrícolas.

\footnotetext{
${ }^{8}$ La serpiente Chicchan pierde sus cascabeles cuando las Pléyades desaparecen del cielo durante el período de un mes de su conjunción, en mayo (Milbrath, 1999: figura 7.3).
} 


\section{BIBLIOGRAFÍA}

Aveni, Anthony F.

1999 "Astronomy in the Mexican Codex Borgia", Archaeoastronomy 24. Supplement to the Journal of the History of Astronomy, 30: S1-S20.

Bricker, Harvey M. y Victoria R. Bricker

2011 Astronomy in the Maya Codices. Philadelphia: American Philosophical Society.

2015 "Linearity and Cyclicity in Precolumbian Time Reckoning", The Measure and Meaning of Time in Mesoamerica and the Andes, pp. 165-182, Anthony F. Aveni (ed.). Washington, D.C.: Dumbarton Oaks.

Bricker, Victoria R.

"A Method for Dating Venus Almanacs in the Borgia Codex", Archaeoastronomy 26. Supplement to the Journal of the History of Astronomy, 32: S21-S43.

2010 "A comparison of Venus Instruments in the Borgia and Madrid Codices", Astronomers, Scribes and Priests, pp. 309-332, Gabrielle Vail and Christine Hernández (eds.). Washington, D.C.: Dumbarton Oaks.

Espenak, Fred and Jean Meeus

2006 Five Millenium Canon of Solar Eclipses: -1999 to +3000 . Hanover: NASA Center for AeroSpace Information.

Hernández, Christine and Gabrielle Vail

2010 "A Case for Scribal Interaction: Evidence from the Madrid and Borgia Codices," Astronomers, Scribes and Priests, pp. 333-364, Gabrielle Vail and Christine Hernández (eds.). Washington, D.C.: Dumbarton Oaks.

Just, Brian R.

2004 "In Extenso Almanacs in the Madrid Codex", The Madrid Codex: New Approaches to Understanding an Ancient Maya Manuscript, pp. 255-276, Gabrielle Vail and Anthony F. Aveni (eds.). Boulder: University Press of Colorado.

Lange, Rainer and Noel M. Swerlow

2006 Planetary, Lunar and Stellar Visibility. 3.1.0 Alycone Software, <http://www.alcyone.de. $>$.

Leopold, Aldo Starker

1959 Wildlife of Mexico. Berkeley: University of California Press.

Milbrath, Susan

1999 Star Gods of the Maya: Astronomy in Art, Folklore and Calendars. Austin: University of Texas Press.

2013 Heaven and Earth in Ancient Mexico: Astronomy and Seasonal Cycles in the Codex Borgia. Austin: University of Texas Press. 
Šprajc, Ivan

1993 "The Venus-rain-maize Complex in the Mesoamerican World View: Part I", Journal for the History of Astronomy, 24: 17-70.

1996 Venus, lluvia y maíz: simbolismo y astronomía en la cosmovisión mesoamericana. México: Instituto Nacional de Antropología e Historia (Serie Arqueología).

Taube, Karl

1992 The Major Gods of Ancient Yucatan. Washington, D.C.: Dumbarton Oaks (Studies in Pre-Columbian Art and Archaeology, 32).

Terán, Silvia y Christian H. Rasmusen

1994 La milpa de los mayas: la agricultura de los mayas prehispánicos y actuales en el noreste de Yucatán. Mérida: Danida.

Thompson, J. Eric S.

1960 Maya Hieroglyphic Writing: An Introduction. Norman: University of Oklahoma Press.

Tozzer, Alfred M.

1941 Landa's "Relación de las Cosas de Yucatán": A Translation. Cambridge: The Peabody Museum (Papers of the Peabody Museum of Archaeology and Ethnology, Harvard University, 18).

Vail, Gabrielle and Anthony F. Aveni (eds.)

2004 The Madrid Codex: New Approaches to Understanding an Ancient Maya Manuscript. Boulder: University Press of Colorado.

Vail, Gabrielle and Victoria R. Bricker

2004 "Haab Dates in the Madrid Codex", The Madrid Codex: New Approaches to Understanding an Ancient Maya Manuscript, pp. 171-214, Gabrielle Vail and Anthony F. Aveni (eds.). Boulder: University Press of Colorado.

Verbelen, Felix

Solar Eclipses in Mesoamerica, AD 1 to $1600,<$ http://user.online.be/felixverbelen>. 


\begin{tabular}{|c|c|c|c|c|}
\hline \multicolumn{5}{|c|}{$\begin{array}{l}\text { Siglo xiv: ciclos de Venus en años de eclipses emparejados } \\
\text { que fueron visibles en Yucatán con una magnitud superior a } 0.50\end{array}$} \\
\hline Venus & Eclipse & Tipo & $\begin{array}{c}\text { Visibilidad en Yucatán } \\
\text { (magnitud) }\end{array}$ & Fecha + página \\
\hline $\begin{array}{c}\text { M-última } \\
3 \text { de sept. de } 1301\end{array}$ & $\begin{array}{l}9 \text { de feb. } \\
\text { de } 1301\end{array}$ & A & $10: 39(0.90)$ & $\begin{array}{l}9 \text { Chicchan } \\
\text { [8 Mol] }\end{array}$ \\
\hline $\begin{array}{c}\text { V-primera } \\
16 \text { de nov. de } 1301\end{array}$ & $\begin{array}{l}5 \text { de ago. } \\
\text { de } 1301\end{array}$ & $\mathrm{~T}$ & no fue visible & $\begin{array}{c}4 \mathrm{lk} \\
\text { [5 Kayab] }\end{array}$ \\
\hline $\begin{array}{c}\text { M-última } \\
29 \text { de mar. de } 1303\end{array}$ & $\begin{array}{l}15 \text { de jun. } \\
\text { de } 1303\end{array}$ & $\mathrm{H}$ & $19: 13(0.92)$ & $\begin{array}{c}7 \text { Imix } \\
\text { [14 Kankin] (p. 17b) }\end{array}$ \\
\hline $\begin{array}{c}\text { V-primera } \\
22 \text { de jun. de } 1303\end{array}$ & $\begin{array}{l}9 \text { de dic. } \\
\text { de } 1303\end{array}$ & $\mathrm{H}$ & no fue visible & $\begin{array}{l}2 \text { Edznab } \\
\text { [6 Tzec] }\end{array}$ \\
\hline $\begin{array}{c}\text { M-última } \\
26 \text { de mar. de } 1311\end{array}$ & $\begin{array}{l}20 \text { de ene. } \\
\text { de } 311\end{array}$ & A & no fue visible & $\begin{array}{l}1 \text { Caban } \\
\text { [10 Yaxkin] }\end{array}$ \\
\hline $\begin{array}{c}\text { V-primera } \\
19 \text { de jun. de } 1311\end{array}$ & $\begin{array}{l}16 \text { de jul. } \\
\text { de } 1311\end{array}$ & $\mathrm{~T}$ & $11: 26(0.97)$ & $\begin{array}{c}9 \text { Ix } \\
{[7 \text { Pax] }}\end{array}$ \\
\hline $\begin{array}{c}\text { M-última } \\
19 \text { de jun. de } 1314 \\
\end{array}$ & $\begin{array}{c}15 \text { de mayo } \\
\text { de } 1314\end{array}$ & A & no fue visible & $\begin{array}{c}3 \text { Lamat } \\
\text { [6 Mac] (p. 17b) }\end{array}$ \\
\hline $\begin{array}{c}\text { V-primera } \\
1 \text { de sept. de } 1314\end{array}$ & $\begin{array}{l}8 \text { de nov. } \\
\text { de } 1314\end{array}$ & $\mathrm{~T}$ & $6: 20(0.67)$ & $\begin{array}{l}11 \text { Chicchan } \\
\text { [18 Zip] }\end{array}$ \\
\hline $\begin{array}{c}\text { V-última } \\
12 \text { de jul. de } 1318\end{array}$ & $\begin{array}{l}3 \text { de mar. } \\
\text { de } 1318\end{array}$ & $\mathrm{H}$ & no fue visible & $\begin{array}{c}13 \mathrm{Cib} \\
{[14 \mathrm{Ch} \text { 'en] }}\end{array}$ \\
\hline $\begin{array}{l}\text { M-primera } \\
29 \text { de jul. de } 1318\end{array}$ & $\begin{array}{l}26 \text { de ago. } \\
\text { de } 1318\end{array}$ & $\mathrm{H}$ & 12:07 (0.99) & $\begin{array}{c}7 \mathrm{~Eb} \\
{[10 \text { Cumku] (p. 13b) }}\end{array}$ \\
\hline $\begin{array}{c}\text { M-última } \\
27 \text { de ago. de } 1325\end{array}$ & $\begin{array}{l}13 \text { de abr. } \\
\text { de } 1325\end{array}$ & $\mathrm{~T}$ & $11: 19(0.90)$ & $\begin{array}{c}11 \mathrm{Ix} \\
{[17 \mathrm{Zac}]}\end{array}$ \\
\hline $\begin{array}{c}\text { V-primera } \\
9 \text { de nov. de } 1325\end{array}$ & $\begin{array}{l}7 \text { de oct. } \\
\text { de } 1325\end{array}$ & A & no fue visible & $\begin{array}{c}6 \text { Chuen } \\
\text { [9 Uo] (p. 13b) }\end{array}$ \\
\hline $\begin{array}{c}\text { V-última } \\
10 \text { de jul. de } 1326\end{array}$ & $\begin{array}{l}3 \text { de abr. } \\
\text { de } 1326\end{array}$ & $\mathrm{~T}$ & no fue visible & $\begin{array}{c}2 \text { Muluc } \\
\text { [7 Zac] (p. 12b) }\end{array}$ \\
\hline $\begin{array}{c}\text { M-primera } \\
27 \text { de jul. de } 1326\end{array}$ & $\begin{array}{l}26 \text { de sept. } \\
\text { de } 1326\end{array}$ & A & $18.15(0.71)$ & $\begin{array}{l}9 \text { Chicchan } \\
\text { [18 Pop] }\end{array}$ \\
\hline $\begin{array}{c}\text { V-primera } \\
19 \text { de ene. de } 329\end{array}$ & $\begin{array}{l}30 \text { de ene. } \\
\text { de } 1329\end{array}$ & A & $16: 22(0.78)$ & $\begin{array}{c}8 \mathrm{Ik} \\
{[5 \mathrm{Mol}] \text { (p. 17b) }}\end{array}$ \\
\hline $\begin{array}{c}\text { V-última } \\
16 \text { de sept. de } 1329\end{array}$ & $\begin{array}{l}7 \text { de jul. } \\
\text { de } 1329\end{array}$ & $\mathrm{~T}$ & no fue visible & $\begin{array}{c}3 \text { Ahau } \\
\text { [3 Kayab] (p. 12b) }\end{array}$ \\
\hline $\begin{array}{c}\text { V-primera } \\
31 \text { de mar. de } 1340\end{array}$ & $\begin{array}{l}25 \text { de jun. } \\
\text { de } 1340\end{array}$ & A & $13: 38(0.66)$ & $\begin{array}{c}12 \text { Cimi } \\
\text { [14 Muan] }\end{array}$ \\
\hline $\begin{array}{c}\text { V-última } \\
4 \text { de dic. de } 1340\end{array}$ & $\begin{array}{l}19 \text { de dic. } \\
\text { de } 1340\end{array}$ & $\mathrm{~T}$ & $11: 21(0.59)$ & $\begin{array}{l}7 \text { Akbal } \\
\text { [6 Xul] }\end{array}$ \\
\hline $\begin{array}{c}\text { M-última } \\
11 \text { de ene. de } 1348\end{array}$ & $\begin{array}{l}31 \text { de ene. } \\
\text { de } 1348\end{array}$ & $\mathrm{H}$ & no fue visible & $\begin{array}{c}6 \mathrm{lk} \\
{[10 \mathrm{Mol}]}\end{array}$ \\
\hline $\begin{array}{c}\text { V-primera } \\
28 \text { de mar. de } 1348\end{array}$ & $\begin{array}{l}26 \text { de jul. } \\
\text { de } 1348\end{array}$ & $\mathrm{H}$ & 18:03 (0.55) & $\begin{array}{c}1 \text { Cauac } \\
\text { [7 Kayab] }\end{array}$ \\
\hline $\begin{array}{c}\text { M-última } \\
14 \text { de mar. de } 1351\end{array}$ & $\begin{array}{l}25 \text { de mayo } \\
\text { de } 1351\end{array}$ & $\mathrm{H}$ & $16: 23(0.58)$ & $\begin{array}{c}7 \mathrm{~Eb} \\
\text { [5 Kankin] (p. 13b) }\end{array}$ \\
\hline
\end{tabular}




\begin{tabular}{|c|c|c|c|c|}
\hline Venus & Eclipse & Tipo & $\begin{array}{c}\text { Visibilidad en Yucatán } \\
\text { (magnitud) }\end{array}$ & Fecha + página \\
\hline $\begin{array}{c}\text { V-primera } \\
7 \text { de jun. de } 1351\end{array}$ & $\begin{array}{l}19 \text { de nov. } \\
\text { de } 1351\end{array}$ & A & no fue visible & $\begin{array}{c}3 \text { Oc } \\
\text { [18 Zotz] (p. 12b) }\end{array}$ \\
\hline $\begin{array}{c}\text { V-última } \\
26 \text { de abr. de } 1355\end{array}$ & $\begin{array}{l}14 \text { de mar. } \\
\text { de } 1355\end{array}$ & A & 9:20 (0.94) & $\begin{array}{c}5 \text { Imix } \\
{[14 \text { Yax] }}\end{array}$ \\
\hline $\begin{array}{c}\text { M-primera } \\
9 \text { de mayo de } 1355\end{array}$ & $\begin{array}{l}7 \text { de sept. } \\
\text { de } 1355\end{array}$ & $\mathrm{~T}$ & no fue visible & $\begin{array}{l}13 \text { Edznab } \\
\text { [6 Pop] }\end{array}$ \\
\hline $\begin{array}{c}\text { M-última } \\
15 \text { de ago. de } 1365\end{array}$ & $\begin{array}{l}21 \text { de feb. } \\
\text { de } 1365\end{array}$ & A & no fue visible & $\begin{array}{c}10 \text { Ben } \\
{[16 \text { Chen] }}\end{array}$ \\
\hline $\begin{array}{c}\text { V-primera } \\
29 \text { de oct. de } 1365\end{array}$ & $\begin{array}{l}17 \text { de ago. } \\
\text { de } 1365\end{array}$ & $\mathrm{~T}$ & $9: 25(0.62)$ & $\begin{array}{c}5 \text { Oc } \\
\text { [13 Cumku] (p. 13b) }\end{array}$ \\
\hline $\begin{array}{c}\text { M-primera } \\
19 \text { de feb. de } 1368\end{array}$ & $\begin{array}{l}15 \text { de jun. } \\
\text { de } 1368\end{array}$ & A & no fue visible & $\begin{array}{c}11 \text { Akbal } \\
\text { [11 Muan] (p. 12b) }\end{array}$ \\
\hline $\begin{array}{c}\text { M-última } \\
26 \text { de oct. de } 1368\end{array}$ & $\begin{array}{l}10 \text { de dic. } \\
\text { de } 1368\end{array}$ & $\mathrm{H}$ & $18: 39(0.55)$ & 7 Imix 4 Xul (p. 17b) \\
\hline $\begin{array}{c}\text { V-primera } \\
21 \text { de mar. de } 1372\end{array}$ & $\begin{array}{l}4 \text { de abr. } \\
\text { de } 1372\end{array}$ & $\mathrm{H}$ & no fue visible & $\begin{array}{c}9 \mathrm{~Eb} \\
{[0 \mathrm{Ceh}]}\end{array}$ \\
\hline $\begin{array}{c}\text { V-última } \\
24 \text { de nov. de } 1372\end{array}$ & $\begin{array}{l}27 \text { de sept. } \\
\text { de } 1372\end{array}$ & $\mathrm{H}$ & 11:07 (0.93) & $\begin{array}{l}3 \text { Lamat } \\
\text { [11 Uo] (p. 13b) }\end{array}$ \\
\hline $\begin{array}{c}\text { M-última } \\
5 \text { de mar. de } 1383\end{array}$ & $\begin{array}{l}4 \text { de mar. } \\
\text { de } 1383\end{array}$ & A & $14: 30(0.90)$ & $\begin{array}{c}4 \text { Edznab } \\
\text { [11 Yax] (p. 17b) }\end{array}$ \\
\hline $\begin{array}{c}\text { V-primera } \\
29 \text { de mayo de } 1383\end{array}$ & $\begin{array}{l}29 \text { de ago. } \\
\text { de } 1383\end{array}$ & $\mathrm{~T}$ & no fue visible & 13 Cib [4 Pax] \\
\hline $\begin{array}{c}\text { M-última } \\
22 \text { de mayo de } 1394\end{array}$ & $\begin{array}{l}1 \text { de feb. } \\
\text { de } 1395\end{array}$ & $\mathrm{~T}$ & no fue visible & $\begin{array}{c}13 \text { Chicchan } \\
\text { [3 Chen] (p. 12b) }\end{array}$ \\
\hline $\begin{array}{c}\text { V-primera } \\
7 \text { de ago. de } 1394\end{array}$ & $\begin{array}{c}28 \text { de jul. } \\
\text { de } 1395\end{array}$ & A & $6: 43(0.60)$ & $\begin{array}{c}8 \mathrm{Ik} \\
\text { [0 Cumku] (p. 17b) }\end{array}$ \\
\hline $\begin{array}{c}\text { M-primera } \\
27 \text { de abr. de } 1395\end{array}$ & $\begin{array}{l}21 \text { de ene. } \\
\text { de } 1395\end{array}$ & $\mathrm{~T}$ & $15: 15(0.63)$ & $\begin{array}{l}3 \text { Cauac } \\
\text { [12 Mol] }\end{array}$ \\
\hline $\begin{array}{c}\text { M-última } \\
30 \text { de dic. de } 1395\end{array}$ & $\begin{array}{l}17 \text { de jul. } \\
\text { de } 1395\end{array}$ & A & $7: 27(0.01)$ & $\begin{array}{c}11 \mathrm{Cib} \\
{[9 \text { Kayab] }}\end{array}$ \\
\hline $\begin{array}{c}\text { M-última } \\
6 \text { de ago. de } 1397\end{array}$ & $\begin{array}{c}26 \text { de mayo } \\
\text { de } 1397\end{array}$ & $\mathrm{~T}$ & $19: 21(0.86)$ & $\begin{array}{c}1 \text { Men } \\
\text { [18 Kankin] (p. 17b) }\end{array}$ \\
\hline $\begin{array}{c}\text { V-primera } \\
19 \text { de oct. de } 1397\end{array}$ & $\begin{array}{l}20 \text { de nov. } \\
\text { de } 1397\end{array}$ & A & no fue visible & $\begin{array}{c}10 \text { Ben } \\
{[11 \text { Tzec] }}\end{array}$ \\
\hline
\end{tabular}

TABLA 1

Las fechas de visibilidad de Venus proceden de Lange y Swerdlow (2006) en la región de Mérida, con $3^{\circ}$ de elevación.

$\mathrm{M}=$ Venus como Estrella de Mañana; $\mathrm{V}=$ Venus como Estrella Vespertina; primera = cuando Venus es visible por primera vez; última = cuando lo es por última vez, antes de la conjunción con el sol.

El tipo y magnitud de eclipses visibles en Yucatán proceden de Espenak y Meeus (op. cit.) y Verbelen (op. cit.), y la hora local se basa en Uxmal (UT - 5:59 horas, según Verbelen).

Tipo de eclipses: $\mathrm{A}=$ anular, $\mathrm{T}=$ total, $\mathrm{H}=$ híbrido, $\mathrm{P}=$ parcial.

Se proporcionan todas las fechas en calendario juliano y las fechas mayas en la correlacion GMT-2 de $584,283$.

Se señalan con negritas las fechas mayas que corresponde a eclipses visibles en Yucatán. Entre paréntesis se anotan las páginas del Códice Madrid correspondientes a fechas del Tzolkin que se encuentran en la misma página. 


\begin{tabular}{|c|c|c|c|c|}
\hline \multicolumn{5}{|c|}{$\begin{array}{l}\text { Siglo xv: ciclos de Venus en años de eclipses emparejados } \\
\text { que fueron visibles en Yucatán con una magnitud más allá de } 0.50\end{array}$} \\
\hline Venus & Eclipse & Tipo & $\begin{array}{l}\text { Visibilidad en Yucatán } \\
\text { (magnitud) }\end{array}$ & Fecha + página \\
\hline $\begin{array}{c}\text { M-última } \\
20 \text { de mayo de } 1402 \\
\end{array}$ & $\begin{array}{l}4 \text { de mar. } \\
\text { de } 1402\end{array}$ & A & no fue visible & $\begin{array}{l}2 \text { Edznab } \\
{[16 \text { Yax }]}\end{array}$ \\
\hline $\begin{array}{c}\text { V-primera } \\
5 \text { de ago. de } 1402\end{array}$ & $\begin{array}{l}28 \text { de ago. } \\
\text { de } 1402\end{array}$ & A & $15: 35(0.97)$ & $\begin{array}{l}10 \text { Men } \\
\text { [8 Pop] }\end{array}$ \\
\hline $\begin{array}{l}\text { M-última } \\
5 \text { de ago. de } 1405\end{array}$ & $\begin{array}{l}1 \text { de ene. } \\
\text { de } 1405\end{array}$ & $\mathrm{H}$ & no fue visible & 9 Eb [15 Yaxkin] \\
\hline \multirow[t]{2}{*}{$\begin{array}{c}\text { V-primera } \\
18 \text { de oct. de } 1405\end{array}$} & $\begin{array}{l}26 \text { de jun. } \\
\text { de } 1405\end{array}$ & $\mathrm{H}$ & $11: 18(1.01)$ & $\begin{array}{l}3 \text { Lamat } \\
\text { [11 Pax] (p. 13b) }\end{array}$ \\
\hline & $\begin{array}{l}21 \text { de dic. } \\
\text { de } 1405\end{array}$ & A & no fue visible & $\begin{array}{c}12 \text { Cimi } \\
\text { [4 Yaxkin] }\end{array}$ \\
\hline $\begin{array}{c}\text { M-primera } \\
4 \text { de feb. de } 1416\end{array}$ & $\begin{array}{l}27 \text { de mayo } \\
\text { de } 1416\end{array}$ & $\mathrm{~T}$ & no fue visible & $\begin{array}{c}13 \mathrm{Cib} \\
{[4 \text { Muan] }}\end{array}$ \\
\hline $\begin{array}{c}\text { M-última } \\
11 \text { de oct. de } 1416\end{array}$ & $\begin{array}{l}19 \text { de nov. } \\
\text { de } 1416\end{array}$ & A & $6: 08(0.87)$ & $\begin{array}{c}7 \mathrm{~Eb} \\
{[15 \mathrm{Tzec}](\mathbf{p} .13 \mathrm{~b})}\end{array}$ \\
\hline $\begin{array}{c}\text { M-última } \\
10 \text { de mayo de } 1426\end{array}$ & $\begin{array}{l}7 \text { de mayo } \\
\text { de } 1426\end{array}$ & $\mathrm{H}$ & no fue visible & $\begin{array}{l}5 \text { Lamat } \\
\text { [6 Kankin] }\end{array}$ \\
\hline $\begin{array}{c}\text { V-primera } \\
28 \text { de jul. de } 1426 \\
\end{array}$ & $\begin{array}{l}30 \text { de oct. } \\
\text { de } 1426\end{array}$ & $\mathrm{H}$ & $12: 16(0.62)$ & $\begin{array}{c}12 \text { Kan } \\
\text { [17 Zotz] (p. 12b) }\end{array}$ \\
\hline $\begin{array}{c}\text { V-última } \\
13 \text { de jun. de } 1430\end{array}$ & $\begin{array}{c}22 \text { de feb. } \\
\text { de } 1430\end{array}$ & $\mathrm{~T}$ & $17: 12(0.69)$ & $\begin{array}{c}1 \text { Men } \\
\text { [13 Yax] (p. 17b) }\end{array}$ \\
\hline $\begin{array}{c}\text { M-primera } \\
29 \text { de jun. de } 1430 \\
\end{array}$ & $\begin{array}{l}19 \text { de ago. } \\
\text { de } 1430\end{array}$ & A & no fue visible & $\begin{array}{l}10 \text { Ben } \\
\text { [6 Pop] }\end{array}$ \\
\hline $\begin{array}{c}\text { M-última } \\
7 \text { de mayo de } 1434\end{array}$ & $\begin{array}{l}7 \text { de jun. } \\
\text { de } 1434\end{array}$ & $\mathrm{~T}$ & no fue visible & $\begin{array}{c}7 \text { Imix } \\
\text { [19 Muan] (p. 17b) }\end{array}$ \\
\hline $\begin{array}{c}\text { V-primera } \\
26 \text { de jul. de } 1434 \\
\end{array}$ & $\begin{array}{l}30 \text { de nov. } \\
\text { de } 1434\end{array}$ & A & $17: 59(0.92)$ & $\begin{array}{l}1 \text { Caban } \\
{[10 \mathrm{Xul}]}\end{array}$ \\
\hline $\begin{array}{c}\text { M-última } \\
26 \text { de jul. de } 1437 \\
\end{array}$ & $\begin{array}{l}5 \text { de abr. } \\
\text { de } 1437\end{array}$ & A & $9: 54(0.86)$ & $\begin{array}{c}13 \text { Ix } \\
\text { [17 Ceh] (p. 17b) } \\
\end{array}$ \\
\hline $\begin{array}{c}\text { V-primera } \\
8 \text { de oct. de } 1437 \\
\end{array}$ & $\begin{array}{l}30 \text { de sept. } \\
\text { de } 1437\end{array}$ & $\mathrm{~T}$ & no fue visible & $\begin{array}{c}9 \mathrm{~Eb} \\
{[10 \mathrm{Zip}]}\end{array}$ \\
\hline $\begin{array}{c}\text { M-última } \\
4 \text { de mayo de } 1442\end{array}$ & $\begin{array}{l}12 \text { de ene. } \\
\text { de } 1442\end{array}$ & A & no fue visible & $\begin{array}{r}1 \text { Caban } \\
{[15 \mathrm{Mol}]}\end{array}$ \\
\hline $\begin{array}{c}\text { V-primera } \\
23 \text { de jul. de } 1442 \\
\end{array}$ & $\begin{array}{l}7 \text { de jul. } \\
\text { de } 1442\end{array}$ & $\mathrm{~T}$ & $17: 31(0.58)$ & $\begin{array}{c}8 \text { Ben } \\
\text { [11 Kayab] (p. 13b) }\end{array}$ \\
\hline $\begin{array}{c}\text { M-primera } \\
9 \text { de abr. de } 1451\end{array}$ & $\begin{array}{c}28 \text { de jun. } \\
\text { de } 1451\end{array}$ & $\mathrm{~T}$ & $17: 41(0.52)$ & $\begin{array}{l}10 \text { Chuen } \\
\text { [4 Kayab] }\end{array}$ \\
\hline $\begin{array}{c}\text { M-última } \\
14 \text { de dic. de } 1451\end{array}$ & $\begin{array}{l}23 \text { de dic. } \\
\text { de } 1451\end{array}$ & A & no fue visible & $\begin{array}{l}6 \text { Muluc } \\
\text { [17 Yaxkin] }\end{array}$ \\
\hline $\begin{array}{c}\text { V-primera } \\
27 \text { de feb. de } 1452\end{array}$ & $\begin{array}{l}17 \text { de jun. } \\
\text { de } 1452\end{array}$ & $\mathrm{~T}$ & 7:59 (0.96) & $\begin{array}{c}1 \mathrm{Cimi} \\
\text { [14 Pax] (p. 13b) }\end{array}$ \\
\hline $\begin{array}{c}\text { V-última } \\
27 \text { de oct de } 1452 \\
\end{array}$ & $\begin{array}{l}11 \text { de dic. } \\
\text { de } 1452\end{array}$ & A & no fue visible & $\begin{array}{c}9 \text { Akbal } \\
\text { [6 Yaxkin] }\end{array}$ \\
\hline $\begin{array}{c}\text { M-primera } \\
24 \text { de ene. de } 1456\end{array}$ & $\begin{array}{l}5 \text { de abr. } \\
\text { de } 1456\end{array}$ & A & no fue visible & $\begin{array}{c}11 \mathrm{Ix} \\
{[2 \mathrm{Mac}]}\end{array}$ \\
\hline
\end{tabular}




\begin{tabular}{|c|c|c|c|c|}
\hline Venus & Eclipse & Tipo & $\begin{array}{l}\text { Visibilidad en Yucatán } \\
\text { (magnitud) }\end{array}$ & Fecha + página \\
\hline $\begin{array}{c}\text { M-última } 28 \\
\text { de sept. de } 1456\end{array}$ & $\begin{array}{l}29 \text { de sept. } \\
\text { de } 1456\end{array}$ & A & $13: 49(0.62)$ & $\begin{array}{l}6 \text { Chuen } \\
\text { [14 Zip] (p. 13b) }\end{array}$ \\
\hline $\begin{array}{c}\text { M-última } \\
\text { de } 26 \text { de abr. de } 1466\end{array}$ & $\begin{array}{l}16 \text { de mar. } \\
\text { de } 1466\end{array}$ & $\mathrm{~T}$ & no fue visible & $\begin{array}{l}3 \mathrm{Cimi} \\
{[4 \mathrm{Ceh}]}\end{array}$ \\
\hline $\begin{array}{c}\text { V-primera } \\
\text { de } 26 \text { de jul. de } 1466\end{array}$ & $\begin{array}{l}9 \text { de sept. } \\
\text { de } 1466\end{array}$ & A & $11: 47(0.72)$ & $\begin{array}{c}11 \text { Akbal } \\
{[16 \text { Uo] (p. 12b) }}\end{array}$ \\
\hline $\begin{array}{c}\text { V-última } \\
1 \text { de jun. de } 1470\end{array}$ & $\begin{array}{l}2 \text { de ene. } \\
\text { de } 1470\end{array}$ & A & no fue visible & $\begin{array}{c}13 \mathrm{~lx} \\
{[12 \mathrm{Mol}](\mathrm{p} .17 \mathrm{~b})}\end{array}$ \\
\hline \multirow[t]{2}{*}{$\begin{array}{c}\text { M-primera } \\
\text { de } 16 \text { de jun. de } 1470\end{array}$} & $\begin{array}{l}28 \text { de jun } \\
\text { de } 1470\end{array}$ & $\mathrm{~T}$ & no fue visible & $\begin{array}{l}8 \text { Chuen } \\
\text { [9 Kayab] }\end{array}$ \\
\hline & $\begin{array}{l}22 \text { de dic. } \\
\text { de } 1470\end{array}$ & A & 7:03 (0.54) & $\begin{array}{c}3 \text { Lamat } \\
{[1 \mathrm{Mol}] \text { (p. 13b) }}\end{array}$ \\
\hline $\begin{array}{c}\text { M-última } \\
14 \text { de jul. de } 1477\end{array}$ & $\begin{array}{l}13 \text { de feb. } \\
\text { de } 1477\end{array}$ & $\mathrm{H}$ & $14: 50(0.99)$ & $\begin{array}{c}12 \text { Ben } \\
{[16 \text { Yax] (p. 17b) }}\end{array}$ \\
\hline $\begin{array}{c}\text { V-primera } \\
25 \text { de sept. de } 1477 \\
\end{array}$ & $\begin{array}{l}8 \text { de ago. } \\
\text { de } 1477\end{array}$ & $\mathrm{~T}$ & no fue visible & $\begin{array}{l}6 \text { Muluc } \\
\text { [7 Pop] }\end{array}$ \\
\hline $\begin{array}{c}\text { V-última } \\
\text { de } 30 \text { de mayo de } 1478\end{array}$ & $\begin{array}{l}3 \text { de feb. } \\
\text { de } 1478\end{array}$ & A & no fue visible & $\begin{array}{l}3 \text { Lamat } \\
\text { [6 Yax] (p. 13b) }\end{array}$ \\
\hline $\begin{array}{c}\text { M-primera } \\
14 \text { de jun. de } 1478\end{array}$ & $\begin{array}{l}29 \text { de jul. } \\
\text { de } 1478\end{array}$ & $\mathrm{~T}$ & $5: 12(0.60)$ & $\begin{array}{l}10 \text { Kan } \\
\text { [2 Uayeb] }\end{array}$ \\
\hline $\begin{array}{c}\text { M-primera } \\
\text { de } 17 \text { de ene. de } 1480\end{array}$ & $\begin{array}{l}8 \text { de jun. } \\
\text { de } 1480\end{array}$ & $\mathrm{H}$ & no fue visible & $\begin{array}{c}1 \mathrm{Kan} \\
{[12 \mathrm{Pax}]}\end{array}$ \\
\hline $\begin{array}{c}\text { M-última } \\
\text { de } 21 \text { de sept. de } 1480\end{array}$ & $\begin{array}{l}1 \text { de dic. } \\
\text { de } 1480\end{array}$ & $\mathrm{H}$ & $15: 40(0.58)$ & $\begin{array}{c}8 \text { Ahau } \\
\text { [3 Yaxkin] (p. 12b) }\end{array}$ \\
\hline $\begin{array}{c}\text { V-última } \\
\text { de } 4 \text { ago. de } 1481\end{array}$ & $\begin{array}{l}28 \text { de mayo } \\
\text { de } 1481\end{array}$ & A & $9: 37(0.92)$ & $\begin{array}{c}4 \text { Edznab } \\
{[1 \text { Pax] (p.17b) }}\end{array}$ \\
\hline $\begin{array}{c}\text { M-primera } \\
22 \text { de ago. de } 1481\end{array}$ & $\begin{array}{l}21 \text { de nov. } \\
\text { de } 1481\end{array}$ & $\mathrm{~T}$ & no fue visible & $\begin{array}{l}12 \mathrm{Men} \\
{[13 \mathrm{Xul}]}\end{array}$ \\
\hline $\begin{array}{c}\text { V-primera } \\
\text { de } 17 \text { de feb. de } 1484\end{array}$ & $\begin{array}{l}26 \text { de mar. } \\
\text { de } 1484\end{array}$ & $\mathrm{~T}$ & $17: 33(0.59)$ & $\begin{array}{l}10 \text { Chuen } \\
\text { [19 Ceh] }\end{array}$ \\
\hline $\begin{array}{c}\text { V-última } \\
\text { de } 17 \text { de oct. de } 1484\end{array}$ & $\begin{array}{l}20 \text { de sept. } \\
\text { de } 1484\end{array}$ & A & no fue visible & $\begin{array}{l}6 \text { Muluc } \\
\text { [12 Zip] }\end{array}$ \\
\hline $\begin{array}{c}\text { M-primera } \\
28 \text { de mar. de } 1491\end{array}$ & $\begin{array}{c}8 \text { de mayo } \\
\text { de } 1491\end{array}$ & A & $5: 21(0.77)$ & $\begin{array}{c}9 \text { Oc } \\
{[3 \text { Muan] }}\end{array}$ \\
\hline $\begin{array}{c}\text { M-última } \\
3 \text { de dic. de } 1491 \\
\end{array}$ & $\begin{array}{l}2 \text { de nov. } \\
\text { de } 1491\end{array}$ & $\mathrm{~T}$ & no fue visible & $\begin{array}{l}5 \text { Lamat } \\
\text { [16 Tzec] }\end{array}$ \\
\hline $\begin{array}{c}\text { V-primera } \\
14 \text { de feb. de } 1492\end{array}$ & $\begin{array}{l}26 \text { de abr. } \\
\text { de } 1492\end{array}$ & A & $12: 15(0.59)$ & $\begin{array}{c}12 \mathrm{Kan} \\
\text { [12 Kankin] (p. 12b) }\end{array}$ \\
\hline $\begin{array}{c}\text { V-última } \\
13 \text { de oct. } 1492\end{array}$ & $\begin{array}{l}21 \text { de oct. } \\
\text { de } 1492\end{array}$ & A & no fue visible & $\begin{array}{c}8 \mathrm{lk} \\
{[5 \text { Tzec] (p. 17b) }}\end{array}$ \\
\hline $\begin{array}{c}\text { M-primera } 13 \\
\text { de ene. de } 1496\end{array}$ & $\begin{array}{l}14 \text { de feb. } \\
\text { de } 1496\end{array}$ & A & no fue visible & $\begin{array}{l}10 \mathrm{Ben} \\
{[1 \mathrm{Zac}]}\end{array}$ \\
\hline $\begin{array}{c}\text { M-última } \\
17 \text { de sept. de } 1496\end{array}$ & $\begin{array}{l}8 \text { de ago. } \\
\text { de } 1496\end{array}$ & $\mathrm{~T}$ & $15: 47$ (0.96) & $\begin{array}{c}4 \text { Muluc } \\
\text { [12 Pop] (p. 13b) }\end{array}$ \\
\hline
\end{tabular}




\begin{tabular}{|c|c|c|c|c|}
\hline Venus & Eclipse & Tipo & $\begin{array}{l}\text { Visibilidad en Yucatán } \\
\text { (magnitud) }\end{array}$ & Fecha + página \\
\hline $\begin{array}{c}\text { M-última } \\
\text { de 11de abr. } 1506\end{array}$ & $\begin{array}{l}24 \text { de ene. } \\
\text { de } 1506\end{array}$ & A & no fue visible & $\begin{array}{l}3 \text { Chicchan } \\
\text { [3 Yax] (p. 12b) }\end{array}$ \\
\hline $\begin{array}{c}\text { V-primera } \\
4 \text { de jul. de } 1506\end{array}$ & $\begin{array}{l}20 \text { de jul. } \\
\text { de } 1506\end{array}$ & $\mathrm{~T}$ & $5: 55(0.61)$ & $\begin{array}{c}10 \mathrm{Ik} \\
\text { [0 Uayeb] (p. 12b) }\end{array}$ \\
\hline $\begin{array}{c}\text { V-primera } \\
\text { de } 8 \text { de feb. de } 1508\end{array}$ & $\begin{array}{l}2 \text { de ene. } \\
\text { de } 1508\end{array}$ & A & $8: 40(0.51)$ & $\begin{array}{c}8 \text { Ben } \\
\text { [1 Chen] (p. 13b) }\end{array}$ \\
\hline $\begin{array}{c}\text { V-última } \\
7 \text { de oct. de } 1508\end{array}$ & $\begin{array}{l}29 \text { de mayo } \\
\text { de } 1508\end{array}$ & $P$ & no fue visible & $\begin{array}{l}13 \text { Imix } \\
{[9 \text { Pax }]}\end{array}$ \\
\hline $\begin{array}{c}\text { M-primera } \\
26 \text { de oct. de } 1508\end{array}$ & $\begin{array}{l}28 \text { de jun } \\
\text { de } 1508\end{array}$ & $\mathrm{P}$ & no fue visible & $\begin{array}{c}4 \text { Chuen } \\
\text { [19 Kayab] (p. 12b) }\end{array}$ \\
\hline $\begin{array}{c}\text { V-primera } \\
6 \text { de feb. de } 1516\end{array}$ & $\begin{array}{l}4 \text { de ene. } \\
\text { de } 1516\end{array}$ & $\mathrm{P}$ & no fue visible & $\begin{array}{l}7 \text { Caban } 5 \text { Chen } \\
\text { (p. 13b) }\end{array}$ \\
\hline $\begin{array}{c}\text { V-última } \\
5 \text { de oct. de } 1516\end{array}$ & $\begin{array}{l}30 \text { de jun. } \\
\text { de } 1516\end{array}$ & A & no fue visible & $\begin{array}{l}3 \text { Men } 3 \text { Cumku } \\
\text { (p. 12b) }\end{array}$ \\
\hline $\begin{array}{c}\text { M-primera } \\
23 \text { de oct. de } 1516\end{array}$ & $\begin{array}{l}23 \text { de dic. } \\
\text { de } 1516\end{array}$ & $\mathrm{~T}$ & $6: 19(0.82)$ & $\begin{array}{l}10 \text { Chuen } \\
{[14 \mathrm{Mol}]}\end{array}$ \\
\hline
\end{tabular}

TABLA 2

Las fechas de visibilidad de Venus proceden de Lange y Swerdlow (2006) en la región de Mérida, con $3^{\circ}$ de elevación.

$\mathrm{M}=$ Venus como Estrella de Mañana; $\mathrm{V}=$ Venus como Estrella Vespertina; primera = cuando Venus es visible por primera vez; última = cuando lo es por última vez, antes de la conjunción con el sol.

El tipo y magnitud de eclipses visibles en Yucatán proceden de Espenak y Meeus (op. cit.) y Verbelen (op. cit.), y la hora local se basa en Uxmal (UT - 5:59 horas, según Verbelen).

Tipo de eclipses: $\mathrm{A}=$ anular, $\mathrm{T}=$ total, $\mathrm{H}=$ híbrido, $\mathrm{P}=$ parcial.

Se proporcionan todas las fechas en calendario juliano y las fechas mayas en la correlacion GMT-2 de $584,283$.

Se señalan con negritas las fechas mayas que corresponde a eclipses visibles en Yucatán. Entre paréntesis se anotan las páginas del Códice Madrid correspondientes a fechas del Tzolkin que se encuentran en la misma página. 